Session 2525

\title{
Teaching Teaming Through Spacecraft Design
}

\author{
Dr. Rachel Shinn, Dr. Ronald Madler \\ Embry Riddle Aeronautical University, Prescott, AZ
}

\begin{abstract}
Project teamwork is essential to the spacecraft design process - in industry as well as in the classroom. At our university, engineering seniors begin the spacecraft design courses having some project teamwork experience, but mostly for short duration projects. We instructors have found that our students don't necessarily possess the skills required to be productive members of a larger engineering team for an entire semester. To help the students work better in teams during the spacecraft design experience, we have implemented a series of exercises and structures. This paper outlines the tools we have used to enable teaming, the grading strategies we have implemented and the interdisciplinary aspects of our design teams.
\end{abstract}

\section{Introduction}

Because of the complexity of spacecraft design, with its many subsystems, teaming is an essential part of our design courses. We cannot take for granted that students will know how to operate in a team environment and we must enable students to learn the teaming process. Students need both guidance and structure to enable them to work effectively in teams.

In this paper, we will present various tools we have used to teach teaming to our senior design students. These tools address the issues of giving the students support and guidance, providing a framework for teaming, handling under-performing team members and better communication with instructors. We also present the grading strategy we have used since grading team projects can be challenging. Because our spacecraft design projects, by their nature, involve aspects from various disciplines, we also present the interdisciplinary aspects of our design teams.

II. Tools for Teaming

Background

We began our experience working with teams in the fall of 1999. As novices, we assigned teams and hoped that the teams would work well together with minimal guidance. Some teams worked 
well and some were less successful, but overall, the teams worked well together with little instructor mediation. We had the teams select a team leader and required that each team assign each member to a particular spacecraft subsystem. They did a fairly good job of distributing and responsibly doing the work. Overall, we were quite lucky that these teams managed to work out their difficulties on their own and be effective.

In the later years, we were not as lucky and discovered are as where teams needed more support and guidance. We noticed the need to enable students to develop rapport within the team and for them to be able to implement respectful communication. We needed an effective means of handling ineffective team members. That is, team members who simply would not participate or who interacted with the team in a destructive way. Finally, we needed to give the students a framework for teaming that could guide them in how to be an effective team.

We implemented the following six tools that helped address these issues with teaming: Building rapport within the team; Lectures on teaming practices; Development of a Code of Cooperation; Development of a Conflict Resolution Plan; Use of a team logbook; Weekly team leader meetings with the instructor.

\section{Building rapport within the team}

An observation we had from fall 99 was that the teams that had the greatest success working together met once a week in an informal way, sometimes over a meal. This helped the students to feel more comfortable working together and to express their concerns to the group in an honest, yet respectful manner. So, for the fall of 2000, we started the year with a hotdog and hamburger barbecue on campus for the class, in an attempt to help the students get to know each other before the grueling work of the semester began. This helped to break the ice, somewhat. Unfortunately, some of the students with poor social skills did not interact with their classmates; in retrospect, icebreakers prior to the barbecue would have been helpful to make the informal gettogether more effective. In addition to icebreakers, the introduction of a teaming structure and code of cooperation helped them derive a set of agreements about how to interact with each other.

\section{Lectures on teaming practices}

We gave a series of 2 lectures based on work by a colleague teaching design at our Daytona Beach campus ${ }^{1}$. In these lectures, we discussed the roles that must be distributed amongst the team members, what it means to be a team, effective communication, goal specification, task distribution, consensus building, what an effective team looks like, what an ineffective team looks like, how to listen, how to give constructive feedback, individual accountability, and the importance of group interdependence.

Team roles that we gave the students were leader, recorder, timekeeper and gatekeeper. These roles correspond to: running the meeting; recording important notes and decisions of the meeting; being sure the time is respected and ensuring that the agenda is followed; that the team keeps on track and that all members are participating. 
In order for the group to be a team, they must be clear on their common goal and be able to respect the contributions of their fellow team members. The team must have a meeting structure that focuses on what questions need to be answered, assigning team members to be responsible for specific tasks, reporting on the status of these tasks to determine if the question has been answered and if not, what the proper question needs to be. This structure enables the students to have an agenda and a direction to follow. It also creates personal responsibility, which sometimes can get lost when students do group projects.

Communication is also a key area in which students need some direction. We cover how to listen effectively and how to respond constructively. Responses should not point fingers directly at another team member. They should rather emphasize the individual's personal response to the topic at hand.

\section{Code of Cooperation}

The code of cooperation is the set of rules that the team members agree to abide by for the duration of the semester. This sets the norms of behavior that are acceptable for interactions within the team. It must be visible to all team members during all team meetings, and each team member should have their own copy, kept in their individual journal. We had the students put this signed document in the front of their logbook. Elements of the code of cooperation should be behaviors that help team members see their successes, find ways to make ideas work, keep every team member focused on how to positively work towards the goal rather than focusing on what is not working, enable team members to win and help the team have fun in the process. In other words, the code of cooperation should help the team to avoid destructive behaviors and encourage behaviors that are constructive to the team.

At the very start of the course, the students were given examples of codes of cooperation and were instructed to write their own code of cooperation that would include the rules they wanted to work by. This enabled them to think about how they were going to work as a team.

An example of a code of cooperation follows:

\section{Code of Conduct}

\section{WILL:}

1. Attend meetings regularly.

2. Listen and show respect to all members.

3. Always put forth my best effort and fair share of work on the design project

4. Not waste time by being stuck on the same problem for more than 20 min. Ask someone. (If at any point during the design process, I am having trouble, I will seek immediate help from the lead engineer, another team member or the instructor.)

5. Pay attention when others are talking...be respectful.

6. Share all ideas regardless of how insignificant I think they may be.

7. Listen to and give constructive criticism.

8. Allow one person at a time to speak during discussions relative to the project. 
9. Not make assumptions about team members or assignments of tasks.

10. Be on time.

11. Let someone know if I am going to be late.

12. Not engage in side conversations (weekend plans, sports, etc.) until the official meeting time is over.

13. Help provide a relaxed and well-nourished learning environment.

14. Have fun!

15. Create an agenda prior to planned meeting and post via email to all team members.

\section{Conflict Resolution Plan}

The students are also required to prepare a conflict resolution plan in which they outline the procedures to be taken in the event there is a conflict within the team. It needs to be clear that failure to resolve issues within the team reflects poorly on the team and that in extreme cases, a student may be "fired" (which means either removal from the team and/or failing the course). The conflict resolution plan is meant to help them, early on, decide how they will handle breaches of their code of cooperation. An example conflict resolution plan follows:

Conflict Resolution:

1. All problems and tardiness will be documented.

2. If a problem occurs, the issue will be identified.

3. The team shall confront the issue and those involved and then document the issue and sign.

4. The team shall then have a meeting and talk about it, identifying changes that will ensure it will not happen again.

5. The proposed changes will be documented and signed.

6. If the issue persists, the instructor will be notified and shown all appropriate documentation.

This conflict resolution plan was implemented. In one case, it resulted in several meetings with a team and the instructors and finally resulted in a student being removed from the team. After this, the team worked effectively together.

\section{Logbook}

This idea for the logbook came from a colleague at our sister campus in Florida ${ }^{1}$. The team logbook is required for all teams and provides a record of teaming, both in and out of class. It is a record of all meetings, discussions, assigned activities/tasks and their time frame, team decisions, and planned activities. The appendix of the logbook contains all team assignments and team graded work. The appendix also contains a record of all assigned tasks (and who the task is assigned to) and the status of those tasks. The logbooks were available in class to be examined by the instructor at any time. The logbook becomes part of the permanent record of the course. In addition, the logbook contains the Code of Cooperation and the Conflict Resolution Plan, mentioned above. A more detailed explanation of the structure of the logbook follows. 
Each team meeting or activity is recorded as a single entry in the logbook with the pages numbered in sequence and dated. The team leader, recorder and timekeeper sign the last page of each entry as witnesses that the information included is accurate. The logbook consists of a 3 ring binder so that pages can be easily added. Each entry should be started on a new page and should contain the minutes of the meeting or activity and any decisions that have been made. These should include who was present, the meeting agenda, notes on discussions and decisions and who took responsibility of what, the schedule of planned activities and the next meeting time.

The logbook had an index so that relevant information can be found quickly.

The appendices contain task tracking, graded work, reference material, and any other material deemed appropriate (this could include relevant websites, contact people, etc.).

We found that the logbook made communication, as a whole, much better. It was also an effective structure that kept the students work and progress up front for all the team to see as well as allowing the instructor to see how the team was progressing.

\section{Team leader meetings}

Weekly meetings with the team leads enabled both the instructors and the teams to better communicate issues or concerns. These weekly meetings with the team leaders consisted of clarifying assignments, answering any questions about our expectations and the students to bringing forward any concerns about the class. It also gave the team leaders the opportunity to share their experiences with their classmates (the other team leaders) and proved to be a very good means of communication between instructors and students. We have been using this since the fall of 1999.

We should note that while all these tools were presented to the students, the degree to which they used them effectively varied from team to team.

\section{Grading}

Grading team projects is challenging. In our (senior) preliminary design course, we have half of the grade based on individual assignments and half on group assignments. In the (senior) detail course, however, the entire grade is based on group work. Ultimately, we must assign each individual a grade, so we devised a method of using team evaluations, individual defenses and our observations to determine the individual grade based on the team grade.

We assign a grade to the team for each item to be graded. There are four team evaluations spaced throughout the semester. In these evaluations, the team members are able to rate and comment on the contribution of all the team members for that portion of the semester. The results of these evaluations are then weighted against the team's average evaluation of itself, and this is used to increase or decrease the individual's grade from the group grade. For instance, if the team grade was 83 , and an individual on the team was rated 8 out of 10 by his/her team, and the team's overall average rating (average of all the team members scores) was 7.5, then this team 
member would receive a grade of $8 / 7.5 * 83$,or 89 . This allows the team to value exceptional performance and discount poor performance on the team.

Initially, we chose ten categories for students to rate their fellow team members on. The categories were: attendance and punctuality; preparation and organization; information gathering; interpersonal skills; leadership skills; ideas contributed; participation; writing; overall quality of work; overall quantity of work. These categories were meant to cover the various areas in which students were performing. The value in assessing all these categories is that we, the instructors, can readily see how the team members view each other's work. In particular, if a team member is rated low, we can investigate whether that student is truly participating and contributing or not.

On the down side, we noticed two main issues. The first is the case where a team chooses to grade all team members high. In this case, there is no differentiation between team members even when our observations or perusing of the logbook would indicate otherwise. When the team members do this, it generally renders the evaluation tool useless. The second issue is the ability of the instructor to effectively utilize the large amount of data that comes in. In a class of 28 students, the amount of data entry becomes quite large and impractical $-28 \times 10 \times 7=1,960$ data points. A computerized system would make this scheme reasonable to implement.

On the positive side, the data gives an average estimate of the team's perception of each individual performance. This can be communicated back to individuals so that they can see what areas they need to improve.

The number of categories was reduced in later evaluations and the categories modified. This has yet to be assessed. Also, a system of a finite number of bonus point s has been implemented, but the results of this have not yet been assessed. This system, used in addition to the category evaluations, has each team member distribute a fixed number of points amongst the team members so that if each team member is contributing equally, this is reflected in the distribution of the points. If, however a team member is contributing extraordinarily or poorly, this also should be reflected in the distribution of the points. While this point distribution scheme has not been assessed in this particular class, a professor in the Electrical Engineering department here at Embry-Riddle has used it successfully.

The last assessment tool we used to assess individual performance on the team was the use of an individual defense of each team members' area(s). These lasted 45 minutes per person and took an entire week to complete. Each student signed up for a time slot in which they were prepared to present the part of the spacecraft they worked on and defend their work orally to a panel of three faculty members. Because the teams were interdisciplinary, we had representation from Electrical Engineering and/or Computer Engineering on the panel. While this was very time consuming, it gave us the clearest assessment of the student's contributions to the project. We could clearly tell the depth to which they had delved into their subsystem, whether they understood how their subsystem worked with the entire spacecraft and whether or not they had thought through the appropriateness of their design solution. This was, by far, the clearest assessment tool for individual performance. However, it also clearly took a large amount of 
faculty resources.

\section{Early Teaming Experiences}

As our space option evolves, we have added more space courses to support our design sequence. One of these courses is a laboratory course in which the students do experiments related to spacecraft. Because we are a small school, this gave us the opportunity to arrange a group project as a final project for the lab course that gives them a preview of the teaming experience. These students, in their junior year, have a team project that helps them practice teaming with the same students they will be working with in their senior year. This has proved helpful, not only to provide us a preview of potential team conflicts, but also, any teaming they can do before the capstone course is helpful - the more, the better. Learning to work as a team is truly a learning experience for them and cannot be taken for granted.

\section{Interdisciplinary Teaming}

The senior level preliminary design class involves teams of 6 or 7 students. Each team must design an entire space mission within the semester. The design consists of top-level mission requirements and flows down to subsystem level requirements as they come up with a design concept for their top-level requirements. All of the subsystems of the spacecraft are represented, including orbits, attitude, structure, propulsion, power, thermal, communications, telemetry and command, launch, and payload. Each subsystem must be sized and materials and hardware selected, with prices estimated and finally presented to an audience open to the entire community. Some of these subsystems require knowledge of subjects that our students (AE) are only introduced to, such as power and communications.

Because our aerospace engineering (AE) students have just a cursory knowledge of some of these subsystems, we have opened our design class to electrical engineering (EE) and computer engineering (CE) students and have had great success with interdisciplinary teams sharing their expertise. While the EE and CE students have a steep learning curve to understand all the different spacecraft subsystems, they have been valuable contributors to their teams. This has been especially true in the power, communications and telemetry subsystems where EE training is indispensable and computer systems can be chosen based on a much more solid foundation. The AE students have better preparation in the areas of orbits, attitude, structures and propulsion. In order to have the different disciplines work more effectively together on the spacecraft system, we hope to have the EE and CE students take the Fundamentals of Space Systems course with the AE students. In this way, all the students will have equal exposure to the spacecraft subsystems.

The combination of EE, CE and AE students was particularly helpful in the detail design class in which the students design, build, integrate and test their project. The interdisciplinary teams enabled the students to integrate electronic systems that controlled the initiation of various events based on sensor data as well as enabling the students to implement an automated direction control system for motion of their project. 


\section{Conclusions}

In the process of helping students to learn to work effectively in teams, we cannot take for granted what we, as more experienced adults, have learned and use effectively in teams. Students need both guidance and structure to enable them to work effectively in teams.

In this paper, we have presented various tools we have used to teach teaming to our senior design students. These tools include building team rapport, lectures on teaming practices, development of a Code of Cooperation and Conflict resolution plan, use of a team logbook and team leader meetings with the instructors. These tools helped us address the issues of giving the students support and guidance, providing a framework for teaming, handling under-performing team members and better communication with instructors.

Grading team projects is challenging and we have presented one model for doing it. In addition, we have shared our successful experience of integrating other disciplines into our design sequence, as well as one way to give the students a jump-start on the teaming process. Our experiences in teaching the students to work in teams continue to feed our innovation in how to be more effective at enabling teaming.

\section{REFERENCES:}

1. Dr. James Ladesic, Professor of Aerospace Engineering, Embry-Riddle Aeronautical University, conversations and un-published course materials exchanged between 6/01 and 10/01.

\section{DR. RACHEL SHINN}

Dr. Rachel Shinn is an assistant professor of Aerospace Engineering at Embry Riddle Aeronautical University in Prescott, AZ. She received her PhD in Applied Mathematics at the California Institute of Technology in Pasadena, California. Before beginning her teaching career, she spent five years in the space industry, at Hughes Aircraft Co. (currently Boeing), working as a spacecraft systems engineer, with a specialty in spacecraft attitude dynamics.

DR. RONALD MADLER

Dr. Ronald Madler is an associate professor and chair of Aerospace Engineering at EmbryRiddle Aeronautical University in Prescott, AZ. He received his $\mathrm{PhD}$ in Aerospace Engineering Sciences at the University of Colorado, Boulder. 